\title{
Erratum to: Index
}

\section{Erratum to: \\ Index in: P. Barrotta, Scientists, Democracy and Society, Logic, Argumentation \& Reasoning 16 https://doi.org/10.1007/978-3-319-74938-9}

The original version of the index was inadvertently published without the below listed author names:

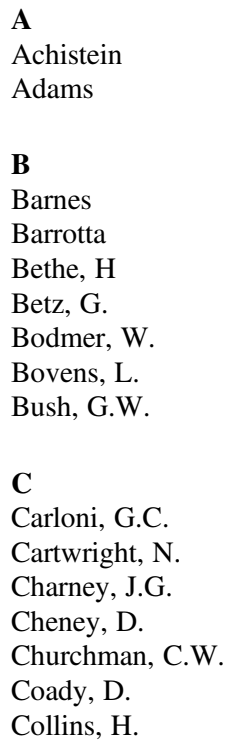


Commager

Conway, E.M.

Corry, R.

D

Darwin, C.

Descates, R.

Dewey, J.

Dorato, M.

Douglas, $\mathrm{H}$.

Douglas, M.

E

Earman, J.

Einstein, A.

Elliott, K.

Evans, R.

F

Feyerabend, P.

G

Gattei, S.

Gervasoni, A.

H

Hansen, J.

Hardie, J.

Hartmann, S.

Hempel, C.G.

Howson, C.

J

James, W.

Jeffrey, R.

John, S.

K

Kitcher, P.

Knorr-Cetina, K.

Kordig, C.R.

Kuhn, T.

L

Lacey, H.

Latour, B.

Lavoisier, A.

Levi, I.

Lomborg, B.

Lovelock, J. 


\section{M}

McMullin, E.

Merlin, T.

Mitchell, S.

Molina, M.

Montuschi, E.

Müller, L.

O

Oppenheimer, R.

Oreskes, N.

P

Palmieri, N.W.

Parker, W.S.

Peirce, C.S.

Phillips. L.D.

Press, S.J.

Preston, J.

Priestley, J.

Putnam, $\mathrm{H}$.

R

Rhodes, R.

Rowland, F.S.

Rudner, R.

S

Schwarz, M.

Semenza, C.

Semenza, E.

Shapin, S.

Shrader-Frechette, K.S.

Solomon, S.

Steele, K.

T

Tanur, J.M.

Teller, E.

Thompson, M.

$\mathbf{U}$

Urbach, P.

W

Wildawsky, A.

Winsberg, E.

Wynne, B. 